\title{
Special issue: Self-assembled structures and materials for accessing new functions
}

\author{
Takeshi Serizawa $^{1} \cdot$ Atsushi Shishido $^{2} \cdot$ Christine K. Luscombe $^{3} \cdot$ Takashi Kato $^{4}$
}

Received: 27 April 2018 / Accepted: 7 May 2018 / Published online: 6 August 2018

(c) The Society of Polymer Science, Japan 2018

The self-assembly of (macro)molecules using various methods (bulk, solution, surface and so on) is a powerful tool for producing functional materials with unique nano- to macroscale structures. Self-assembly can generally be achieved by a low-energy bottom-up process under ambient conditions without any special laboratory equipment. Selfassembled (macro)molecules can show unexpected new properties that are significantly different from the original properties of the individual (macro)molecules. In fact, biological systems effectively utilize (macro)molecular selfassembly in aqueous phases to hierarchically produce complicated structures that are essential for realizing sophisticated biological functions. Even in polymer science for materials development, the concept of self-assembly is being further studied and developed every day. To introduce the latest developments in self-assembled structures and materials with new functions, we organized a hybrid team composed of the Editor-in-Chief, an associate editor,

Takeshi Serizawa

serizawa@ polymer.titech.ac.jp

$\square$ Atsushi Shishido

ashishid@ res.titech.ac.jp

$\bowtie$ Christine K. Luscombe

Luscombeluscombe@uw.edu

$\bowtie$ Takashi Kato

kato@chiral.t.u-tokyo.ac.jp

1 Department of Chemical Science and Engineering, School of Materials and Chemical Technology, Tokyo Institute of Technology, Tokyo, Japan

2 Laboratory for Chemistry and Life Science, Institute of Innovative Research, Tokyo Institute of Technology, Yokohama, Japan

3 Materials Science and Engineering Department, University of Washington, Seattle, WA, United States

4 Department of Chemistry and Biotechnology, School of Engineering, The University of Tokyo, Tokyo, Japan and guest editors to cover a wide range of topics in this special issue.

Since 2012, Polymer Journal has published 8 special issues about pertinent topics in polymer science and related fields [1-8]. In particular, Polymer Journal is celebrating its 50th anniversary this year and published a commemorative issue this January [8]. Following the great successes of previous special issues, we collected 7 Original Articles, 1 Note, 15 Focus Reviews, and 3 Reviews from outstanding researchers from Asia, the United States, and Europe for this issue. These papers will undoubtedly be fascinating and stimulating to the diverse readers of Polymer Journal. We therefore believe that this special collection will contribute to further advances in the self-assembly of polymers. We sincerely appreciate all authors and referees for their contribution to this special issue.

\section{Compliance with ethical standards}

Conflict of interest The authors declare that they have no conflict of interest.

\section{References}

1. Kato T, Aoshima S, Kikuchi H. Special issue: Self-assembled materials. Polym. J. 2012;44:451.

2. Asakura T, Asano A. Preface to the special issue: NMR of Polymers: recent advances and innovations. Polym. J. 2012;44:733.

3. Kanaya T, Sakurai K, Takahara A. Special issue: Application of quantum beams to polymer science and engineering. Polym. J. 2013;45:2.

4. Venanzi M, Kimura S. Special issue: Peptide materials. Polym. J. 2013;45:467.

5. Serizawa T. Special issue: Biorelated polymers and materials. Polym. J. 2014;46:435.

6. Kato T, Kikuchi H, Imai H. Special issue: Fusion materials: creative development of materials and exploration of their function through molecular control. Polym. J. 2015;47:77.

7. Tanaka K, Serizawa T, Chen W-C, Char K, Kato T. Special issue: Polymer surfaces, interfaces and thin films. Polym. J. 2016;48:323.

8. Kato T. 50th volume anniversary of Polymer Journal. Polym. J. 2018;50:1 